Revista Perspectivas Online: Biológicas \& Saúde Julho de 2017, Vol.7, n' 24, p. 64-71

ISSN: 2236-8868 (Online)

DOI: $10.25242 / 886872420171161$

\title{
AVALIAÇÃO DA QUALIDADE DE VIDA EM MULHERES ACOMETIDAS POR CÂNCER DE MAMA EM UMA UNIDADE PARTICULAR, NO MUNICÍPIO DE CAMPOS DOS GOYTACAZES, RJ.
}

\author{
Beatriz Machado Lança Ferreira ${ }^{1}$, Dayana da Silva Malaquias Crespo ${ }^{1}$, Luisa Carvalho Prata, \\ Hillary de Sousa Isabel, Thaís Aparecida de Castro Palermo ${ }^{2}$, Carolina Magalhães dos Santos ${ }^{2}$ \\ \& Eduardo Viana Ricardo ${ }^{3 *}$
}

\begin{abstract}
RESUMO
FERREIRA, B.M.L.; CRESPO, D.S.M.; PRATA, L.C.; ISABEL, H.S.; PALERMO, T.A.C.; SANTOS, C.M. \& RICARDO, E.V. Avaliação da qualidade de vida em mulheres acometidas por câncer de mama em uma unidade particular, no município de Campos dos Goytacazes, RJ. Perspectivas Online: Biológicas e Saúde, v.7, n.24, p. 64-71, 2017.

Segundo o Instituto Nacional do Câncer, o câncer de mama é o tipo de câncer que mais acomete as mulheres em todo o mundo, tanto em países em desenvolvimento quanto em países desenvolvidos. Para o Brasil, em 2014, foram esperados 57.120 casos novos de câncer de mama, com um risco estimado de 56,09 casos a cada 100 mil mulheres, o que corresponde a $20,8 \%$. Com isso os objetivos propostos foram analisar a qualidade de vida de mulheres em tratamento de câncer de mama, identificar fatores correlacionados ao impacto na qualidade de vida vivenciada por estas mulheres e quantificar o índice geral de QV de mulheres em tratamento de câncer de mama. Foi realizada uma

pesquisa de campo em um Hospital de referência ao tratamento de câncer no município de Campos dos Goytacazes, com 29 mulheres com diagnóstico de câncer de mama. A coleta de dados foi feita a partir de um questionário para qualidade de vida (EORTC - QLQ C30 e EORTC - H \& N35), já validados. Os resultados demonstram que o Estado Geral de Saúde atingiu uma média de 73,1 , então podemos considerar que as pacientes consideram sua Qualidade de Vida como satisfatória, visto que aproxima-se do 100 (escore máximo). Desta forma, torna-se evidente a adesão ao tratamento proposto e o impacto de forma positiva, vivenciados por estas pacientes.
\end{abstract}

Descritores: Qualidade de vida; neoplasias malignas da mama; enfermagem oncológica. 


\begin{abstract}
According to the National Cancer Institute, breast cancer is the type of cancer that affects women the world over, both in developing countries and in developed countries. For Brazil, in 2014, 57,120 new cases of breast cancer were expected, with an estimated risk of 56.09 cases per 100,000 women, corresponding to $20.8 \%$. The aim of this study was to analyze the quality of life of women in breast cancer treatment, to identify factors correlated to the impact on quality of life experienced by these women and to quantify the overall QoL index of women undergoing treatment Of breast cancer. A field survey was

carried out at a Hospital for cancer treatment in the municipality of Campos dos Goytacazes, with 29 women diagnosed with breast cancer. Data were collected from a questionnaire for quality of life (EORTC - QLQ C30 and EORTC - H \& N35), already validated. The results show that the General Health Status reached an average of 73.1, so we can consider that the patients consider their Quality of Life to be satisfactory, since it is close to 100 (maximum score). In this way, the adherence to the proposed treatment and the positive impact experienced by these patients becomes evident.
\end{abstract}

Descriptors: Quality of life; breast neoplasms; oncology nursing.

${ }^{1}$ Acadêmicos de Enfermagem - ISECENSA - - Rua Salvador Correa, 139, Centro, Campos dos Goytacazes, RJ, CEP: 28035-310, Brasil;

${ }^{2}$ Institutos Superiores de Ensino do CENSA - ISECENSA - Laboratório de Estudos em Saúde Pública - Rua Salvador Correa, 139, Centro, Campos dos Goytacazes, RJ, CEP: 28035-310, Brasil.

${ }^{3}$ Institutos Superiores de Ensino do CENSA - ISECENSA - Laboratório de Estudos Hospitalares e Terapia Intensiva Rua Salvador Correa, 139, Centro, Campos dos Goytacazes, RJ, CEP: 28035-310, Brasil.

(*) e-mail: enfeduardoviana@hotmail.com

Data da chegada: 18/04/2017 Aceito para publicação: 13/06/2017 


\section{INTRODUÇÃO}

Segundo o Instituto Nacional do Câncer - INCA (2015) o câncer de mama é o tipo de neoplasia que mais acomete mulheres em todo mundo, inclusive no Brasil. A doença causa transformações biopsicossociais que geram profundo impacto na vida dessas pacientes.

A paciente acometida pelo câncer de mama e sua família, vivenciam o diagnóstico como um momento de intensa angústia, sofrimento e ansiedade. Identificado como uma das doenças mais temidas pelas mulheres devido à constância absurda com que vem ocorrendo e, principalmente pelos seus efeitos psicológicos que afetam a imagem pessoal e a sexualidade da mulher que o vivencia, consequentemente sendo devastadora tanto no físico como no psicológico (SOARES, 2009).

A Organização Mundial da Saúde (OMS) define Qualidade de Vida como "a percepção do indivíduo de sua posição na vida, no contexto da cultura e sistemas de valores nos quais vive, em relação aos seus objetivos, expectativas, padrões e preocupações". No entanto, há duas tendências na conceituação do termo: (a) QV entendida de forma mais genérica; (b) QV descrita com relação à saúde. Qualidade de Vida Relacionada à Saúde (QVRS) é um subconjunto do termo amplo QV e recebe esta classificação por incluir os domínios físico, psicológico e social que possuem relação com a saúde (MAJEWSKI et al., 2012).

Assim, a preocupação com o conceito de qualidade de vida $(\mathrm{QV})$ refere-se a um movimento dentro das ciências humanas e biológicas no sentido de valorizar parâmetros mais amplos que o controle de sintomas, como a diminuição da mortalidade ou o aumento da expectativa de vida (MAJEWSKI et al., 2012).

Desta forma o presente estudo tem como objeto avaliar a qualidade de vida, identificar os domínios afetados em mulheres com câncer de mama e caracterizar os dados sóciodemográficos.

\section{METODOLOGIA}

Trata-se de um estudo descritivo-exploratório, com abordagem quantitativa em que foi utilizado o instrumento da European Organization for ResearchandTreatmentofCancerBreastCancer-Specificof Life QuestionnaireQuality Life Questionnaire-30, para avaliação da Qualidade de Vida(Franceschini, 2010). Os domínios contemplados pelo instrumento são: função física, função emocional, função cognitiva, função social, escala de sintomas e estado geral de saúde/qualidade de vida. Cada escore foi transformado numa escala de 0 a 100, de acordo com as diretrizes de utilização do instrumento, onde o zero denota o pior funcionamento e 100 o melhor funcionamento nas escalas funcionais e no Estado Geral de Saúde/Qualidade de Vida, Já na escala de sintomas, quanto maior for o valor do escore apresentado, mais sintomas presentes; porém, quanto menor o valor do escore, menos sintomas. O grupo estudado foram mulheres com diagnóstico recente de neoplasia maligna da mama, em vigência de quimioterapia e /ou radioterapia. Abordadas durante o primeiro ano de tratamento. Foram incluídas mulheres com diagnóstico de câncer de mama, cadastrada na referida instituição e excluídas aquelas com idade inferior a 18 anos e que estivessem mais de um ano de seguimento.

Este estudo foi realizado no ONCOBEDA, em Campos dos Goytacazes/RJ, entre junho a setembro de 2016. Foi submetido ao Comitê de Ética em Pesquisa (CEP) dos Institutos Superiores de Ensino do CENSA, sendo aprovado em 17/06/2016, sob este número de protocolo 56920316.9.0000.5524. Além da aprovação no comitê de ética em pesquisa, este foi realizado mediante a assinatura do Termo de Consentimento Livre e Esclarecido pelos sujeitos, conforme a Resolução 466/12 do Conselho Nacional de Saúde (BRASIL, 2012). A análise estatística foi realizada através do programa Statistical Package for the Social Sciences, versão 19.0. 


\section{RESULTADOS}

O grupo estudado foi composto por 29 mulheres em tratamento para câncer de mama. Apresentaram o seguinte perfil sócio-demográfico: idade média de 55 anos (DP=13,3 ANOS), 41,4\% das pacientes concluíram o ensino médio, $31 \%$ são domésticas, $48,3 \%$ casadas, $28,6 \%$ originárias do município de Campos dos Goytacazes/RJ e com renda familiar per capita média de $\mathrm{R} \$ 604,03$ ( $\mathrm{DP}=\mathrm{R} \$ 414,49)$. Vide tabela 1 a seguir.

Tabela 1: Perfil sociodemográfico de mulheres com CA de mama, de uma unidade hospitalar, no município de Campos dos Goytacazes/RJ.

\begin{tabular}{|c|c|c|}
\hline Variáveis & $\mathbf{n}$ & $\%$ \\
\hline \multicolumn{3}{|l|}{ Idade } \\
\hline De 29 a 48 anos & 7 & 24,1 \\
\hline De 49 a 59 anos & 9 & 31,0 \\
\hline 60 anos ou mais & 13 & 44,8 \\
\hline \multicolumn{3}{|l|}{ Escolaridade } \\
\hline Não frequentou a escola & 1 & 3,4 \\
\hline Ensino Fundamental Incompleto & 5 & 17,2 \\
\hline Ensino Fundamental Completo & 4 & 13,8 \\
\hline Ensino Médio Incompleto & 3 & 10,3 \\
\hline Ensino Médio Incompleto & 12 & 41,4 \\
\hline Ensino Universitário Incompleto & 1 & 3,4 \\
\hline Ensino Universitário Completo & 0 &, 0 \\
\hline \multicolumn{3}{|l|}{ Profissão } \\
\hline Agente Administrativo & 2 & 6,9 \\
\hline Autônoma & 2 & 6,9 \\
\hline Cabeleireira & 2 & 6,9 \\
\hline Contadora & 1 & 3,4 \\
\hline Costureira & 1 & 3,4 \\
\hline Cozinheira & 1 & 3,4 \\
\hline Diarista & 1 & 3,4 \\
\hline Doméstica & 9 & 31,0 \\
\hline Manicure & 2 & 6,9 \\
\hline Modelista & 1 & 3,4 \\
\hline Professora & 3 & 10,3 \\
\hline Recepcionista & 2 & 6,9 \\
\hline Técnica em Enfermagem & 1 & 3,4 \\
\hline Técnica em Edificações & 1 & 3,4 \\
\hline \multicolumn{3}{|l|}{ Estado Civil } \\
\hline Casada & 14 & 48,3 \\
\hline Viúva & 6 & 20,7 \\
\hline Solteira & 4 & 13,8 \\
\hline Divorciada & 5 & 17,2 \\
\hline
\end{tabular}




$\begin{array}{lcc}\text { Região de Procedência } & & \\ \text { Cabo Frio } & 1 & 3,6 \\ \text { Campos dos Goytacazes } & 8 & 28,6 \\ \text { Itaborá́ } & 1 & 3,6 \\ \text { Macaé } & 6 & 21,4 \\ \text { Niterói } & 1 & 3,6 \\ \text { Rio das Ostras } & 1 & 3,6 \\ \text { São Fidélis } & 1 & 3,6 \\ \text { São Franscico de Itabapoana } & 2 & 7,1 \\ \text { São João da Barra } & 3 & 10,7 \\ \text { São Pedro da Aldeia } & 3 & 10,7 \\ \text { Saquarema } & 1 & 3,6\end{array}$

Com relação às características psicométricas do instrumento para a amostra estudada, o coeficiente Alpha de Cronbach foi de 0,92, indicando confiabilidade do instrumento para esta amostra. Esse coeficiente estima a confiabilidade de um questionário aplicado em uma pesquisa. Ele mede a correlação entre respostas em um questionário através da análise das respostas dadas pelos respondentes, apresentando uma correlação média entre as perguntas. $O$ coeficiente $\alpha$ é calculado a partir da variância dos itens individuais e da variância da soma dos itens.

Em relação às escalas funcionais, os itens relacionados a função emocional $(65,0)$ e cognitiva $(50,0)$ e o desempenho de papel $(53,7)$ apresentaram melhores escores. Já os itens função física $(48,5)$ e social $(39,2)$, piores escores. Vide gráfico 1 abaixo.

Gráfico 1: Escala Funcional

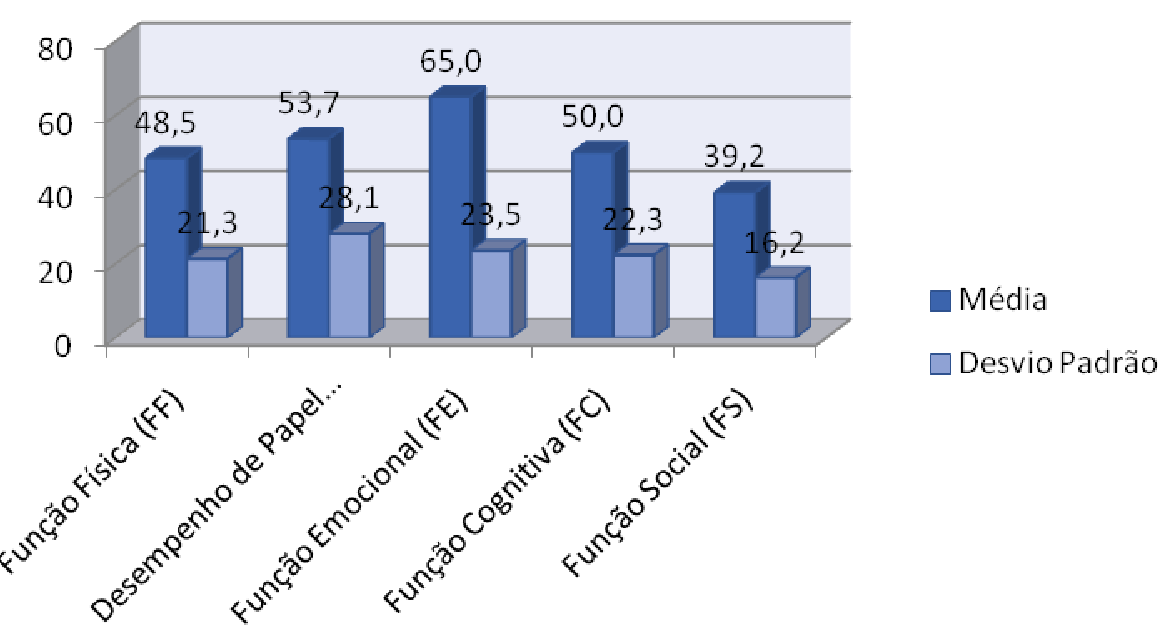

$\mathrm{Na}$ escala de sintomas, os itens predominantes foram fadiga $(56,0)$ e dor $(53,1)$, conforme apresentado no gráfico 2 abaixo. O Estado Geral de Saúde atingiu uma média de 73,1, então podemos considerar que os pacientes consideram sua Qualidade de Vida como satisfatória, visto que aproxima-se do 100 (escore máximo), de acordo com o gráfico 3 abaixo. 
Gráfico 2: Escala de Sintomas

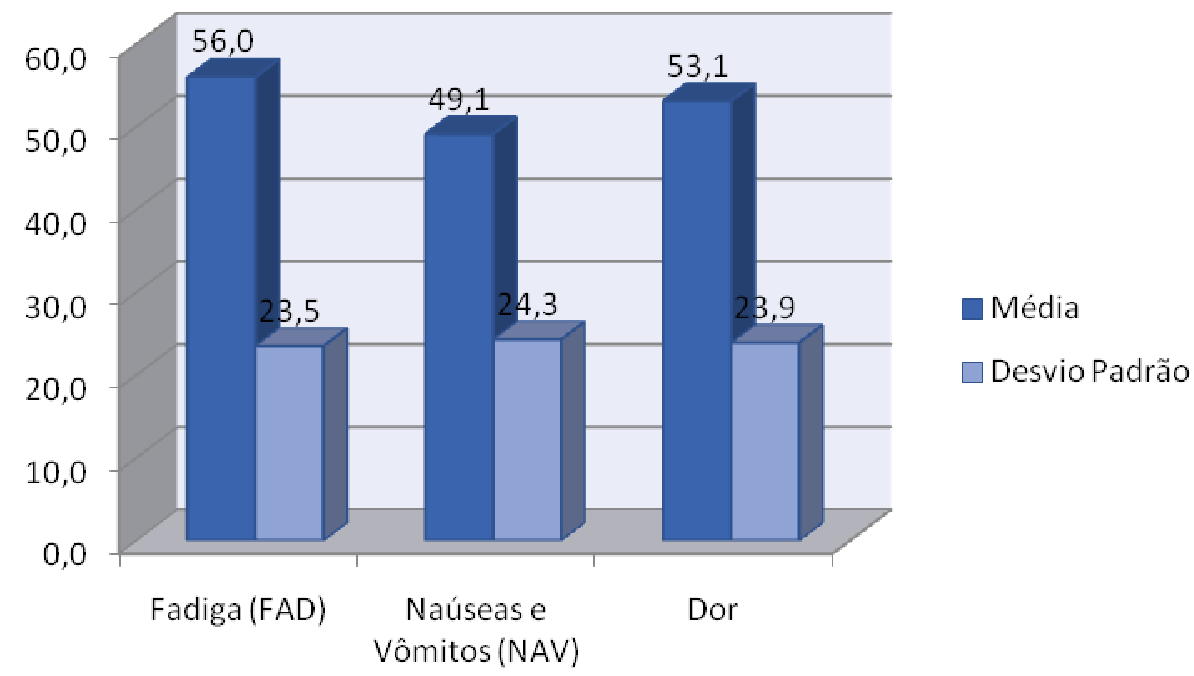

Gráfico 3: Estado Geral de Saúde /Qualidade de Vida (EGS/QV)

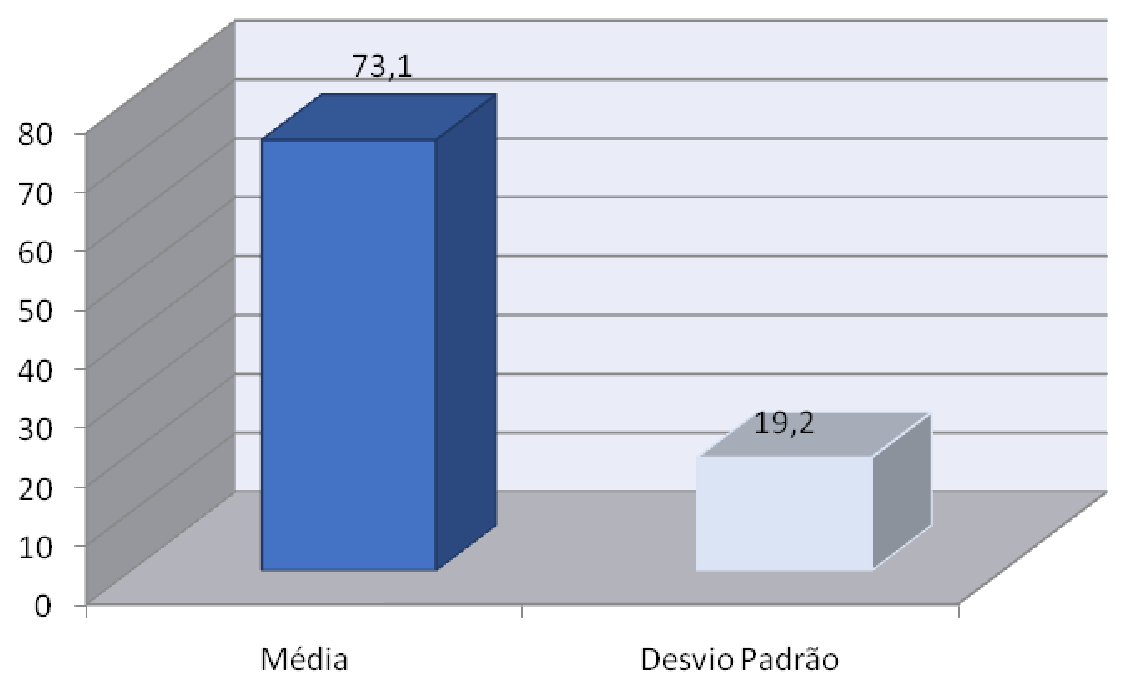

Apesar de serem pacientes do Sistema Único de Saúde, recebem uma assistência diferenciada, o que provavelmente refletiu nos resultados encontrados. Percebemos que os profissionais da referida instituição são capacitados e envolvidos no processo de cuidar, o que pode ter contribuído para manutenção da escala funcional. Estas pacientes também são assistidas por fisioterapeutas, psicólogos e nutricionistas, o que pode ter influenciado no controle dos sintomas. Identificamos também o Grupo Recomeço, onde acontecem encontros semanais com pacientes acometidas por câncer de mama. E para QV, percebemos a influência da consulta com enfermeiro com uma abordagem diferenciada, no sentido da escuta e o toque terapêutico, com finalidade de estimular a manutenção das atividades diárias. 


\section{CONCLUSÕES}

O Estado Geral de Saúde atingiu uma média de 73,1 , então podemos considerar que as pacientes consideram sua Qualidade de Vida como satisfatória, visto que aproxima-se de 100 (escore máximo). Desta forma, torna-se evidente a adesão ao tratamento proposto e o impacto de forma positiva, vivenciados por estas pacientes. Os principais parâmetros empregados na avaliação dos resultados da terapia antineoplásica são a sobrevida livre de doença e a sobrevida global.

Na busca da melhoria da qualidade da assistência a mulheres com câncer da mama, os indicadores de qualidade de vida poderão auxiliar na prática clínica, nortear estratégias de intervenção terapêutica, avaliar sucesso da intervenção após cirurgia e tratamento oncológico, além de criar parâmetros para definição de ações no sentido de promoção de saúde individual ou coletiva. A avaliação da QV foi satisfatória, ressaltando a importância de uma abordagem clínica que valorize a manutenção da boa $\mathrm{QV}$, sendo de extrema importância para o êxito no enfrentamento desta patologia, haja vista que tanto o diagnóstico como o tratamento tendem a deprimir de forma integral o paciente. Considerando essas complexidades, conclui-se que a avaliação da qualidade de vida $(\mathrm{QV})$ torna-se importante auxiliar da prática clínica.

\section{REFERÊNCIAS}

CARVALHO, S. R.; GASTALDO, D;Promoção à saúde e empoderamento: uma reflexão a partir das perspectivas crítico-social pós-estruturalista. Temas Livres. Ciência \& Saúde Coletiva, 13(Sup2):2029-2040, 2008.

FRANCESCHINI, J. et al. Reprodutibilidade da versão em português do Brasil do European Organization for ResearchandTreatmentofCancer Core Qualityof Life Questionnaire em conjunto com seu módulo específico para câncer de pulmão. J. bras.pneumol. v.36, n.5, p 595-602, 2010.

GARBIM, H. B. R.; PEREIRA NETO; A. F.; GUILAM, M. C. R., A internet, o paciente expert e a prática médica: uma análise. Interface: Comunicação, Saúde e Educação, v.12, n. 26. p. 00-00. jul./set. 2008.

INCA. Estimativa 2014: Incidência de câncer no Brasil. Rio de Janeiro INCA, 2014.

INCA. Estimativa 2016: incidência de Câncer no Brasil / Instituto Nacional de Câncer José Alencar Gomes da Silva - Rio de Janeiro: INCA, 2015.

MACHADO, R. R. S.; COSTA, M. C., Disponibilidade da informação para pacientes de câncer: a internet como ferramenta de visibilidade e construção de empoderamento. Cadernos do Tempo Presente, n. 19, mar./abr. 2015, p. 53-66, 2015.

MAJEWSKI, J. M. et al. Qualidade de vida em mulheres submetidas à mastectomia comparada com aquelas que se submeteram à cirurgia conservadora: uma revisão de literatura. Ciência \& Saúde Coletiva, v. 17, n. 3, p.707-716, 2012.

MARQUES, L. F. Qualidade de vida, uma aproximação conceitual .Revista semestral do instituto de psicologia da PUC. Porto Alegre, v.27, p.49-62, jul/dez. 1996

NICOLUSSI, A C.; SAWADA, N O. Qualidade de vida de pacientes com câncer de mama em terapia adjuvante. Revista Gaúcha Enfermagem. Porto Alegre (RS). v. 32, n. 4, p. 759-766, dez 2011.

SOARES, R. G. Aspectos emocionais do câncer de Mama. Sociedade brasileira de psico-oncologia - boletim eletrônico sbpo. Ano IV, ed. 3, jul-ago-set 2009. 
TAVARES, J. S. A.; TRAD, L A B. Estratégias de enfrentamento do câncer de mama: um estudo de caso com famílias de mulheres mastectomizadas. Ciência e Saúde Coletiva, v. 15, n. 1, p. 1349-1358, 2008. 